Original scientific paper

\title{
TOOL STIFFNESS CALCULATION IN ROLL FORMING
}

\author{
Lamprecht, M." \& Leonhartsberger, M. \\ Institute of Production Engineering and Photonic Technologies, Vienna University of Technology, \\ Franz Grill Straße 4, Building 221, 1030 Vienna, Austria \\ E-Mail: lamprecht@ift.at, leonhartsberger@ift.at (\# Corresponding author)
}

\begin{abstract}
In roll forming a metal sheet is incrementally bent at room temperature through numerous pairs of opposing forming rolls. The shafts equipped with rolls represent the most compliant assemblies in the whole roll forming mill. The shafts of one forming pass have to be aligned in the right position, relative to each other and the next as well as the prior forming pass, to avoid defects and to ensure profile dimensions which fulfil the geometric requirements. The forming forces vary greatly, depending on the roll set geometry, settings and stiffness of the individual forming passes. These forming forces lead to deflections of the shafts equipped with rolls, which need to be compensated. In this paper, a finite element model with hexahedron elements of the shafts equipped with rolls is developed, in order to determine its load-deflection behaviour. The accuracy of the model is validated with the aid of experimental data. The provided model shows high accuracy compared to the experimental data and no systematic error over the tested load range is observed. Additionally, the nonlinear stiffness behaviour is investigated in detail to understand the occurring nonlinear effects.

(Received in September 2020, accepted in November 2020. This paper was with the authors 1 week for 1 revision.)
\end{abstract}

Key Words: Roll Forming, Tool Deflection, Stiffness, Deflection Behaviour, Finite Element Method (FEM)

\section{INTRODUCTION}

In roll forming, a certain number of pairs of oppositely arranged shafts with rolls, which are usually different for each forming pass, carry out the bending and determine the shape of the deformed sheet metal [1]. The occurring forces in every individual forming pass vary significantly, depending on the roll sets, the tool stiffness as well as on the geometric positioning of the upper and lower shaft with equipped rolls [2]. Besides these influences of the machine, changes in the material properties lead to the variation of the forming forces [3]. Also, the stiffnesses of the shafts with equipped rolls underly certain variations. To ensure that the produced profile fulfils the tolerance requirements and in order to prevent forming defects, it is necessary that the roll sets are set up in the right positions [4].

The components with the lowest stiffness of the roll forming mill are the upper and lower shafts with equipped rolls. The in-process deflections have to be compensated, which at the moment mainly relies on the experience of the machine operator. Fine adjustments for each forming pass are necessary, which can be very time intensive depending on the machine operator's experience. In order to be able to already compensate for these deflections in the setup process, offside the production plant, adequate models to determine the load-deflection behaviour as well as predictions of realistic loads are needed. If prior to the production of a new profile, the necessary forming forces for each forming pass (determined in finite element, FEsimulations with ideal stiff tools) are known and accurate models to calculate the deflections of the tools are available, the geometry settings for each forming pass can be determined. In consequence, machine downtime and scrap originating from setup process can be reduced, because less adjustments by the machine operator are necessary [2].

Kim and Kim [5] presented the importance of duality in the modelling process for complex systems. As roll forming is such a complex system the presented steps to build up a model for the whole process should be considered. The model presented in this paper represents the 
simulation approach which is used for modelling the presented subsystem of a roll forming line, which is in this case the shafts and tools assembly behaviour under load. The illustrated formalism is already used for a lot of various applications in engineering [6] and might be used for the current problem too.

The deflection behaviour is of high interest when it comes to changing properties of the sheet material. Deviations in strength or thickness of the material change the forces arising in the roll forming process, which inevitably leads to varying deflections of the shafts. In the worst case, these variations cause profiles which do not meet the geometric tolerances anymore. In FE-simulations of the forming process, in numerous studies [7-12] the tools are considered as rigid bodies which are fixed in space. This means that the compliances of the tools are neglected. Abeyrathna et al. [13] used one linear spring for each upper roll set to take the tool stiffnesses into account. Additionally, to the vertically acting spring, Traub et al. [14] used one horizontal spring per forming stand. Müller [15] investigated the differences between measured vertical forces of experimental data with those acquired by numerical simulations for U-shaped cross sections in which the rolls were rigid and fixed in space. As the vertical force levels of the simulations were significantly higher compared to the experiments, he used simple linear springs to account for the stiffness of the tools. Nevertheless, all these approaches are gross simplifications of the real stiffness behaviour. Neither a nonlinear stiffness behaviour nor a deflection curve, which is asymmetric in some cases, were being considered.

In this paper a FE model with hexahedron elements is developed to determine the stiffness behaviour of the shaft of a standard (conventional) roll forming mill with equipped rolls. The FE software ABAQUS was used for all simulations. In a previous study [16] the nonlinear stiffness behaviour of an identical setup was investigated without equipped rolls. The parameters, which significantly influence the nonlinear behaviour, were identified and a two supports Timoshenko beam model with two linear, radially acting springs and one nonlinear rotational spring was developed in order to predict the deflections. The model is valid for shafts without equipped forming rolls. Therefore, a more complex model to depict the stiffening effect due to pretensioned forming rolls is necessary.

The FE simulations were preceded by experiments, which are described in detail by Leonhartsberger et al. [17]. The acquired data from the experiments serve as validation data for the simulation results. Another focus of [17] was the identification of the significant parameters that may change the mechanical characteristics of the setup. The knowledge about parameters which have a significant impact on the stiffness is crucial to build a FE model with adequate system boundaries.

In many fields of engineering, similar mechanical designs can be found, but concerning the stiffness behaviour, they differ substantially from the current problem. Sanchez-Marin et al. [18] developed a model to calculate the deflections of a stepped shaft. Only one loaded part with a different diameter is used and therefore the model cannot be used for the problem of this paper. Radi et al. [19] developed a mathematic model of a shaft-hub press fit, but compared to a loose fit it behaves significantly different. Investigations of the load-deflection behaviour of a shaft with strung, preloaded rolls that have a loose fit and which are supported on both ends could not be found in our literature research.

\section{MATERIALS AND METHODS}

\subsection{Test setup and components of the roll forming mill}

The roll forming mill under investigation and its test setup are shown in Fig. 1. The vertical load, which is generated by infeeding the upper shaft against the lower shaft, is measured with force sensors 8 installed in both forming stands. In order to be able to induce a defined preload force level, a strain gauge-based sensor system (13) is used for the lower shaft. The deflection 
curve of the experimental data is approximated by seven discretely measured points acquired for different rolls. Therefore, axial probes (14) are used. Leonhartsberger et al. [17] identified the main influence parameters amongst the investigated ones on the stiffness behaviour, which are the roll set and the axial preload. This is important to set up an accurate finite element (FE) model.

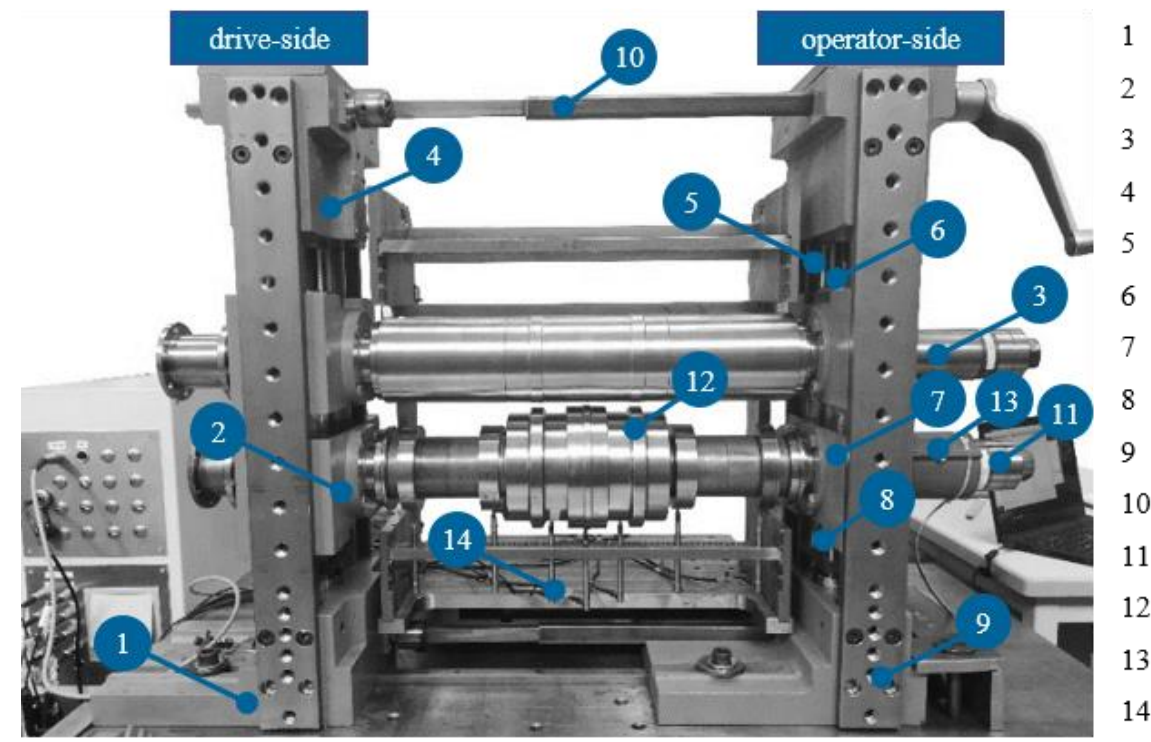

\author{
mill bed mounting \\ bearing pedestal drive-side \\ shaft \\ head part \\ guide post \\ spindle \\ bearing pedestal operator-side \\ force sensor \\ supporting plate \\ connector tube \\ nut \\ forming rolls \\ preload sensor \\ displacement sensors
}

Figure 1: Test setup and components of the roll forming mill.

The three main components of the forming mill are the drive-side stand, the operator-side stand, and the shaft. Among these three components, the shaft with equipped rolls (12) is the most compliant one. The forming stand deformations are the sum of elongation of the posts, compression of the spindles and deformation of the head and mill bed mountings.

The drive-side faces the drivetrain, and during production, at least one shaft of each forming pass is usually connected to the drivetrain. The operator-side stand can be removed easily to equip the shafts with corresponding rolls. Both stands are positioned on the mill bed by a feather key and the mill bed mountings (1) are fixed with screws. To increase the rigidity of the forming stands and to mount some additional parts, the head (4) and the respective mill bed mounting are connected with supporting plates (9). The vertical positioning of the upper (3) and lower shafts is realized by manual spindle drives (6) which are connected to the corresponding bearing pedestal. Normally, the movement of the drive-side pedestal (2) and the movement of the operator-side pedestal (7) are synchronized with a connector tube (10). The bearing pedestals are guided along the posts (5) with a clearance that, due to manufacturing tolerances, ranges between 0.1 and $0.2 \mathrm{~mm}$. For the used forming pass the bore hole diameter of the pedestals and the outer diameters of the guide posts were measured tactilely. Results from these measurements show clearances at the upper limit of the tolerance field. Ergo $0.2 \mathrm{~mm}$ is used in the FE model.

\title{
2.2 Bearing supports of the shaft
}

The bearing supports for the lower shaft are displayed in Fig. 2 as a sectional view. All parts of the assembly are represented in a simplified way. The supports and pedestals of the upper shaft are identical to the lower ones. The drivetrain can be connected at (1). The shaft is three-way supported (see Fig. 2) by one angular contact ball bearing (2) at point A, one needle roller bearing (3) at point B in the bearing pedestal on the drive-side (6), and another identical needle roller bearing (3) at point $\mathrm{C}$ in the bearing pedestal on the operator-side (7). The angular contact 
ball bearing acts as a fixed bearing and the needle roller bearings as floating bearings. Since all bearings take up radial forces, the system is statically overdetermined. This fact results in the support reactions being dependent on the bending stiffness of the shafts with equipped rolls.

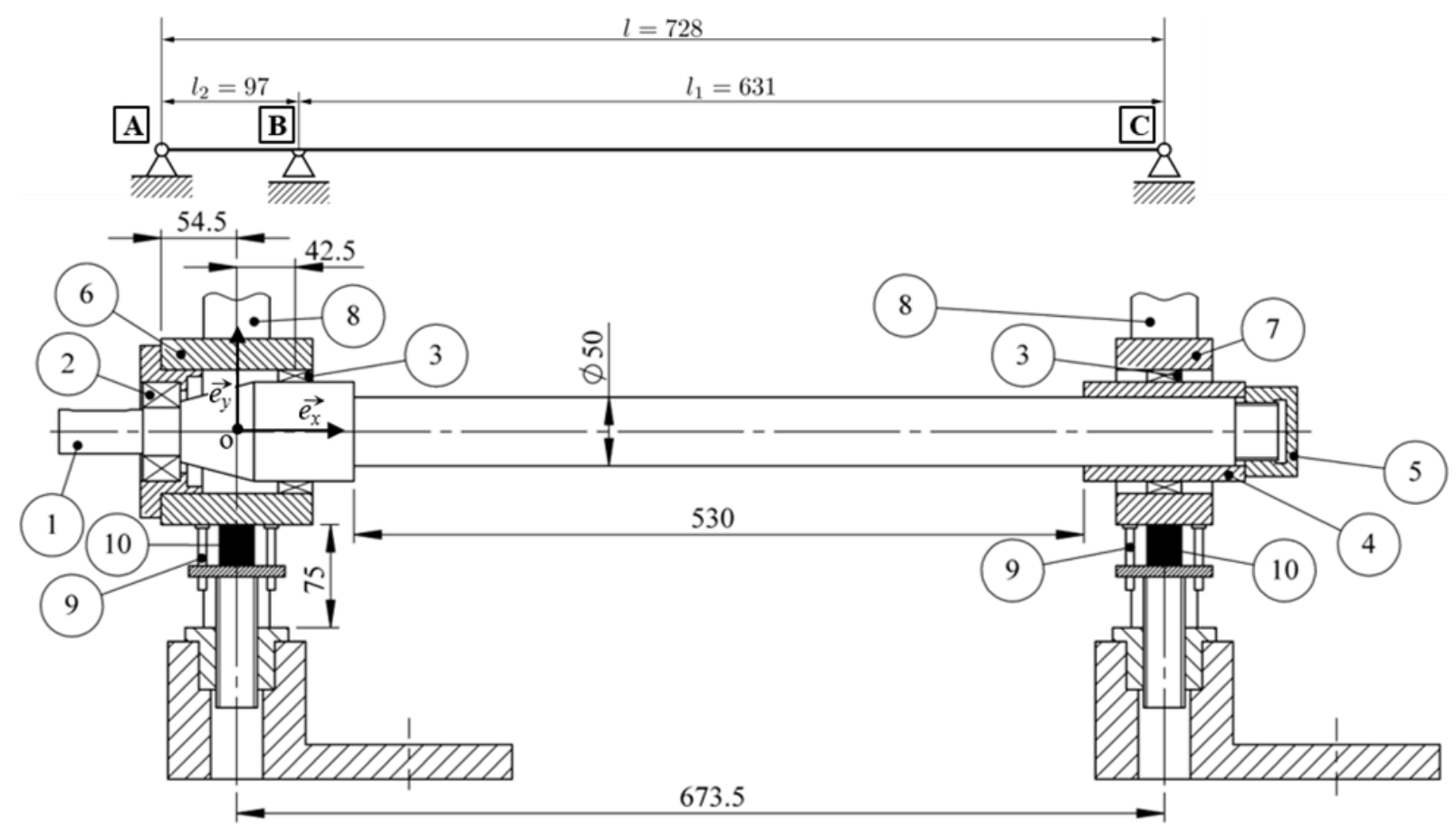

Figure 2: Supports of the lower shaft.

As the bending stiffnesses vary widely (depending on the roll set, preload, clearances, etc.), the support reactions and the force distribution between drive-side and operator-side are different for each setup. The bearing pedestals' axes of rotation in z-direction are located at the horizontal positions of the axes of the posts. Point A and point B on the drive-side have a distance of $54.5 \mathrm{~mm}$ (for A) and $42.5 \mathrm{~mm}$ (for B) to the rotation axis (which is identical to the origin of the coordinate system) of the pedestal. As a result, the drive-side pedestal initiates a relatively high moment into the guide posts 8 compared to the operator sided one. Due to that moment, the posts deflect in $\mathrm{x}$-direction. The greater the deflection of the guide posts, the more the drive-side bearing pedestal tilts, which in turn decreases the overall tool stiffness. The upper shaft's drive-side pedestal causes a moment initiation and deflection of the posts as well. The bearing pedestal of the upper shaft causes an opposing moment into the guide posts, which decreases the maximum deflection of the guide posts, which in turn leads to a decreased deflection of the lower shaft.

In order to determine the deflections of the lower shaft, it is necessary to consider both the upper and lower shaft as well as the drive-side bearing pedestals and the guide posts in a model. By contrast, the force application point of the needle roller bearing on the operator-side is located on the plane of the axis of the guide posts. In the ideal case, no moment is initiated in the posts at this point, except for a possible moment reaction due to the tilting stiffness (only relevant above a certain load level and for small radial clearance of the needle roller bearings). As a result, the posts on the operator-side are not forced to bend. Therefore, the posts and pedestals on the operator-side are not relevant for the overall tool stiffness and can be omitted in a model.

The rolls are positioned between the shaft shoulder on the drive-side and the bearing sleeve (4). By tightening the nut (5), the rolls are pretensioned, which also increases the tool stiffness. Dowel pins (9) keep the force sensors (10) aligned horizontally and prevent the rotation of the mounting plate when the spindle drive is in use. 


\subsection{Introduction of the tool's FE model}

The load-displacement behaviours of the three main components are very different. The forming stands are rather stiff compared to the shafts with equipped rolls and show a linear behaviour over a wide load range. In contrast, the load-deflection behaviour of the shaft with equipped rolls does not show a linear behaviour. In order to calculate the tool stiffness and to keep the calculation effort low, adequate system boundaries have to be defined. The bearing pedestal connection to the spindle drive was set as the system border of the model. In order to acquire the overall deflection of the tools, the calculated displacement values of the FE model simply have to be superimposed by the displacement of the pedestal due to the compliance of the forming stands.

As the geometry is symmetric in respect to the XY-plane, only half of the model is discretized with quadratic hexahedron elements (C3D20, compare [20]), except for the guide post, for which quadratic beam elements are used (3D representation in Fig. 3). The upper and the lower shafts are identical concerning the geometry and supports, but the roll sets are different. As visible in Fig. 3 the drive-side pedestals are part of the assembly and the operatorsided ones are neglected. Point A, B, C in Fig. 3 correspond to A, B, C in Fig. 2. At point C, two reference points (RP1\&RP2) are placed on top of each other. RP1 is fixed in space, which means that all degrees of freedom (1 to 6 ) are locked. RP2 is coupled via a distributed coupling to the corresponding bearing area on the bearing sleeve.

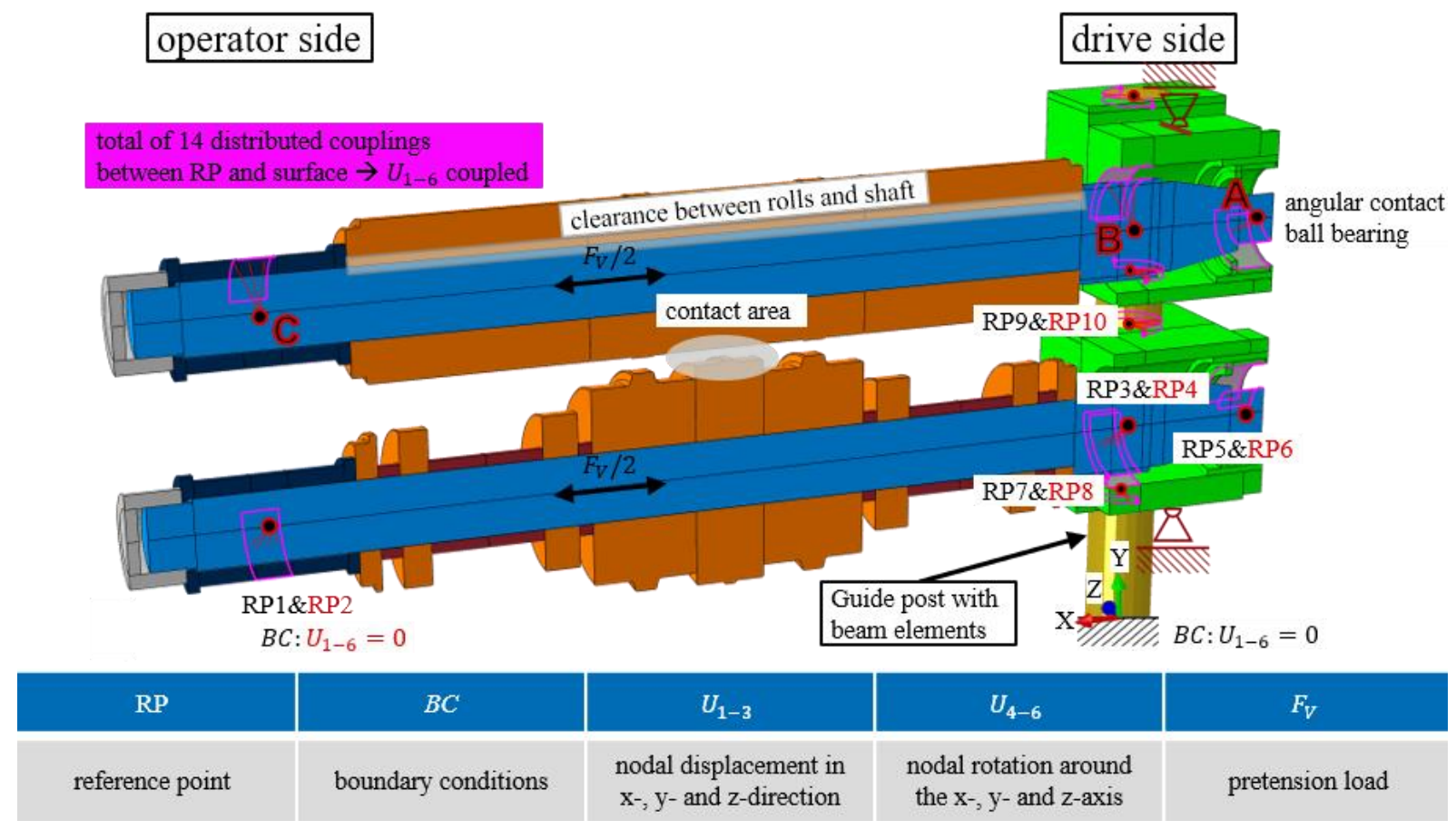

Figure 3: Boundary conditions, bearings and general description of the FE model.

As the rolling elements of the bearings can only transmit compression stresses, only the corresponding half of the bearing area is coupled (the ball bearings used are not pretensioned). In order to determine the load/moment over displacement/rotation behaviour between RP1 and RP2 a connector (see [20]) is used. As B and C are floating bearings, only forces in radial direction can be transmitted. The needle roller bearings as well as the angular contact ball bearings have certain operation clearances: $13.8-65.6 \mu \mathrm{m}$ for the needle bearings and $8-27 \mu \mathrm{m}$ for the ball bearings (data was obtained by experiments). Due to these clearances, the tilting stiffness is zero up to a certain inclination limit. Beyond this limit, the moment over rotation angle curve shows a progressive behaviour. Additionally, the clearances at points A 
and $\mathrm{B}$ in the bearing pedestal on the drive-side allow a limited inclination of the shaft without causing a reaction moment. In order to consider the operation clearances, nonlinear load/moment over displacement/rotation relations between the RPs have to be implemented. In contrary to the points B and C, the RPs at point A are additionally connected in axial direction (fixed support).

The FE calculation is separated in three load steps: pretension, gravity and vertical load. During the first step, the rolls are pretensioned up to a defined value. During the second step, a gravity load is applied. In the last step the upper shaft is moved downwards against the lower shaft in order to induce the loads. Beside the boundary conditions on RP1 at the operator-side, the movement of the bearing pedestal of the lower shaft in vertical direction (y-direction) is prevented at the location of the spindle drive. All other DOFs are not locked, which means, that the pedestal can move in horizontal direction and is able to tilt. The movement of the upper shaft in vertical direction is locked for the first and the second load step. During the last load step the upper shaft is moved against the lower one by applying a certain vertical movement at the boundary condition on the drive-side and operator side.

Due to the clearance between the guide posts and the pedestals and in consequence its tilting, only a small area between these two parts is in contact. The contact face is defined over $180^{\circ}$ and a depth of $15 \mathrm{~mm}$ (from top or bottom face). Distributed couplings with nonlinear connectors (clearances considered) are used between the RP7\&RP8 and RP9\&RP10.

The approximate global mesh size is $3 \mathrm{~mm}$, which results in a total number of about 300000 elements.

As Leonhartsberger et al. [17] found out that the key position is less significant or even inconclusive, the feather key connection is not modelled. Instead, a solid shaft is used and the rolls do not have a groove.

Concerning possible clearance variations, only the extreme cases c-min (all clearances at the lower bound) and c-max (all clearances at the upper bound) are calculated.

For the calculations, three different roll sets for the lower shaft are examined. The roll set of the upper shaft is identical for all setups (see Fig. 4). The range starts from a rather stiff roll set (small tolerances between rolls and shaft, wide rolls, large diameter) and goes to a relatively flexible roll set (many rolls, higher tolerances between rolls and shaft) and the stiffness of moderate roll set lies between them.
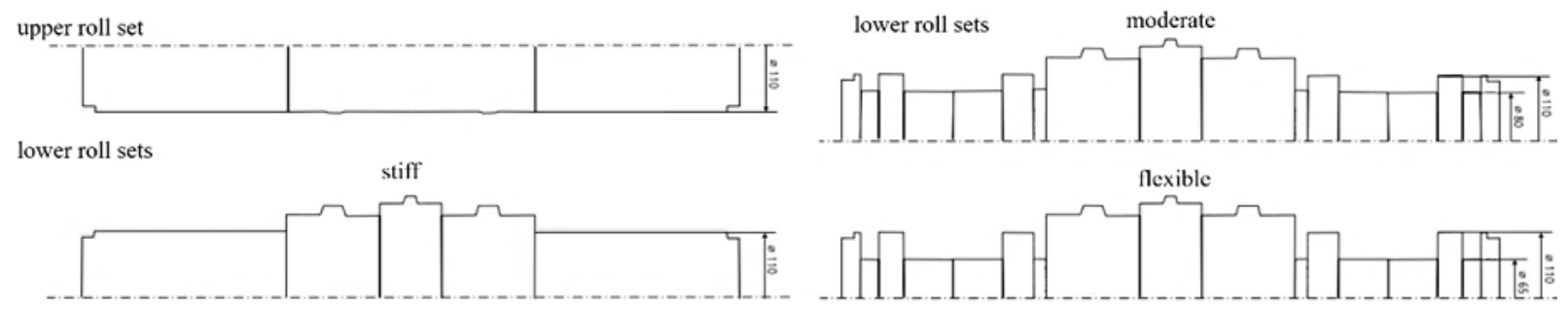

Figure 4: Three different roll sets for investigation.

\section{RESULTS}

The deflection values for a load level of $20 \mathrm{kN}$ (in the last load step) of the FE simulation in comparison to the experimental data are depicted in Fig. 5. The abscissa represents the horizontal position of the shaft and rolls, which is identical to the position in x-direction of the global coordinate system. The ordinate gives the deflection values in negative global ydirection. The dashed lines represent the linear interpolation between the discrete measured displacement values at the seven different positions. The calculated deflection data of the rolls of the lower shaft are displayed as continuous lines with dots at both ends of each roll. Three 
different roll sets (see Fig. 4) are used to evaluate the impact of the accuracy of the simulation model.

The displacement of the forming stands is compensated in all recorded measurement data, which enables a direct comparison with the simulation data. The experimental data is acquired for three different feather key positions: vertical positions either both feather keys are in the tension or compression zone of bending and horizontal position of feather keys. The deflection deviations for different feather key positions increases with decreasing tool stiffness.

A major advantage of the FE model is the possibility to vary the clearances between the rolls and shaft, the operation clearances of the bearings as well as the clearances between the guide posts and the pedestals, independently and targeted, which is not possible for the experiments. The specific coaxiality errors can be incorporated (shift in vertical direction of the rolls), which is not done for the simulations shown in Fig. 5. The outer diameter tolerance of the shaft combined with the inner diameter tolerances of the rolls result in a minimum and a maximum clearance. Only the maximum case c-max is considered in the comparison in Fig. 5. The simulated roll deflections should be located within the different feather key positions of the experimental results. For the stiff and flexible roll set this is the case, but for the moderate roll set the experimental data is more compliant than the simulation with the weakest configuration (maximum of all clearances).

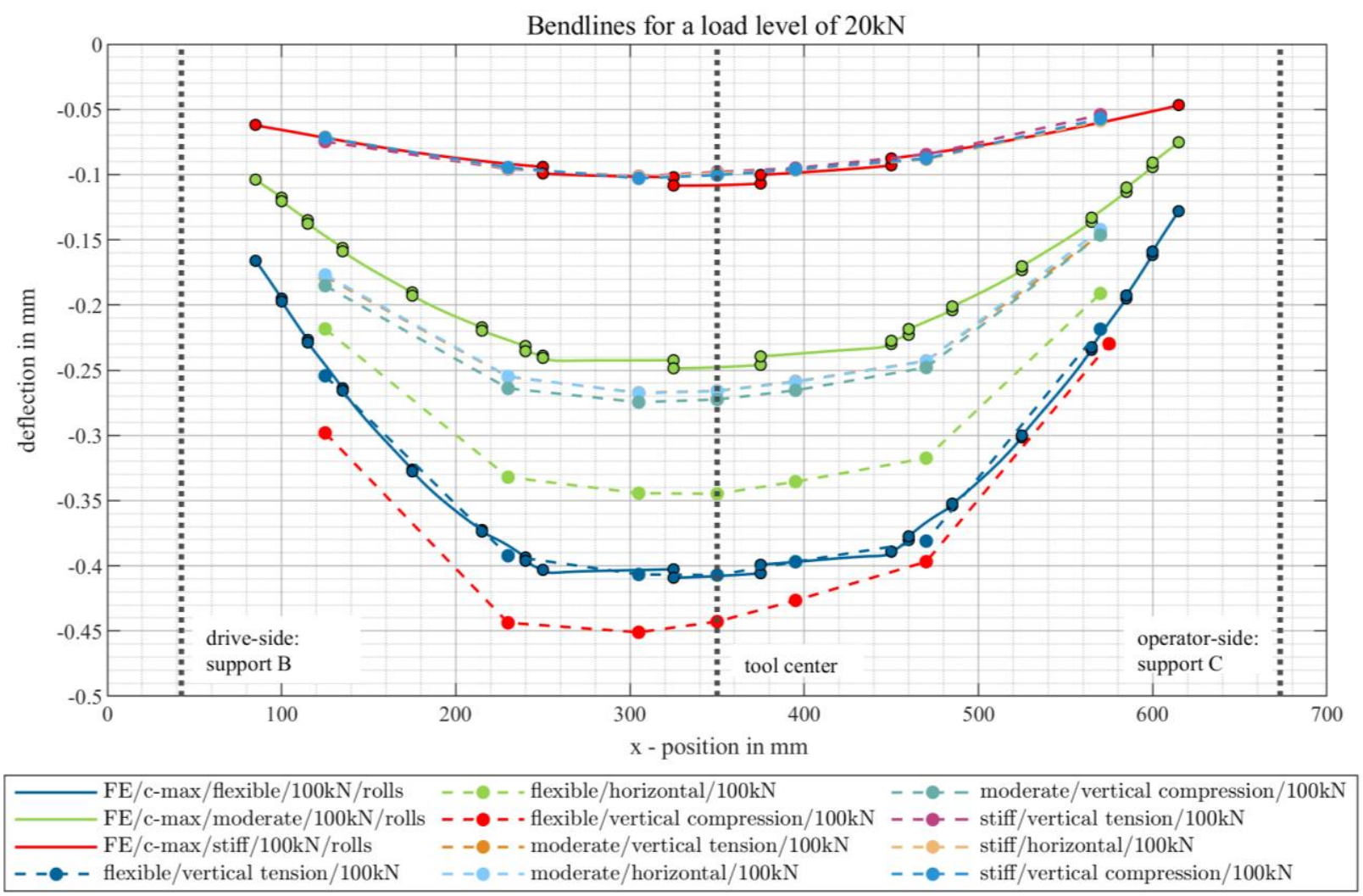

Figure 5: Comparison of deflections between experimental and simulation results.

Leonhartsberger et al. [17] showed that above a certain preload limit value, a further increase in preload does not result in a significant increase of stiffness (valid for the investigated vertical load range of up to $20 \mathrm{kN}$ ). This limit value is in the range of $80 \mathrm{kN}$ up to $120 \mathrm{kN}$ axial preload, which is the reason why the median value of $100 \mathrm{kN}$ is chosen to evaluate the reliability of the simulation models.

In order to be able to make a well-founded statement of the behaviour of the FE model over the entire load range a mean absolute error $(M A E)$ over the whole load range was calculated. The formula for the $M A E_{j}$ is: 


$$
M A E_{j}=\left.\left(\frac{\sum_{i=1}^{n=7}\left(w_{F E_{i}}-w_{M_{i}}\right)}{n}\right)\right|_{F=F[j]}
$$

The deflection values of the experiments $w_{M_{i}}$ are the mean values of all three feather key positions. The differences for the seven discrete measured points (locations of the axial probes) between the calculated deflection values, at the positions $w_{F E_{i}}$ and the experimental results $w_{M_{i}}$ divided by the number of measured points, is calculated for each vertical load level. Fig. 6 displays the $M A E$ development over the entire load range. The flexible, moderate and stiff roll sets with minimum and maximum clearance are considered. The maximum absolute error is $0.06 \mathrm{~mm}$ for the minimum clearance of the flexible roll set. The stiff roll set shows a very low deviation over the load range. The simulation results for the other two roll sets with maximum clearances have a smaller error compared to the minimum ones, which allows the assumption that the clearances in reality are close to the upper limit of the tolerance range. By increasing the load level, the $M A E$ tends to decrease for the moderate as well as for the flexible roll set with minimum clearance. The flexible roll set with maximum clearance shows a very good compliance and no clear deviation tendency between simulation and experiments. According to the result no systematic error of the FE model can be observed in the $M A E$ over load curves, but just three different roll set do not allow a general statement about the reliability of the FE model for a wide range of different roll sets and vertical loads. In future studies, it is necessary to start with a systematic variation of all relevant influence parameters, especially the geometries of the rolls.

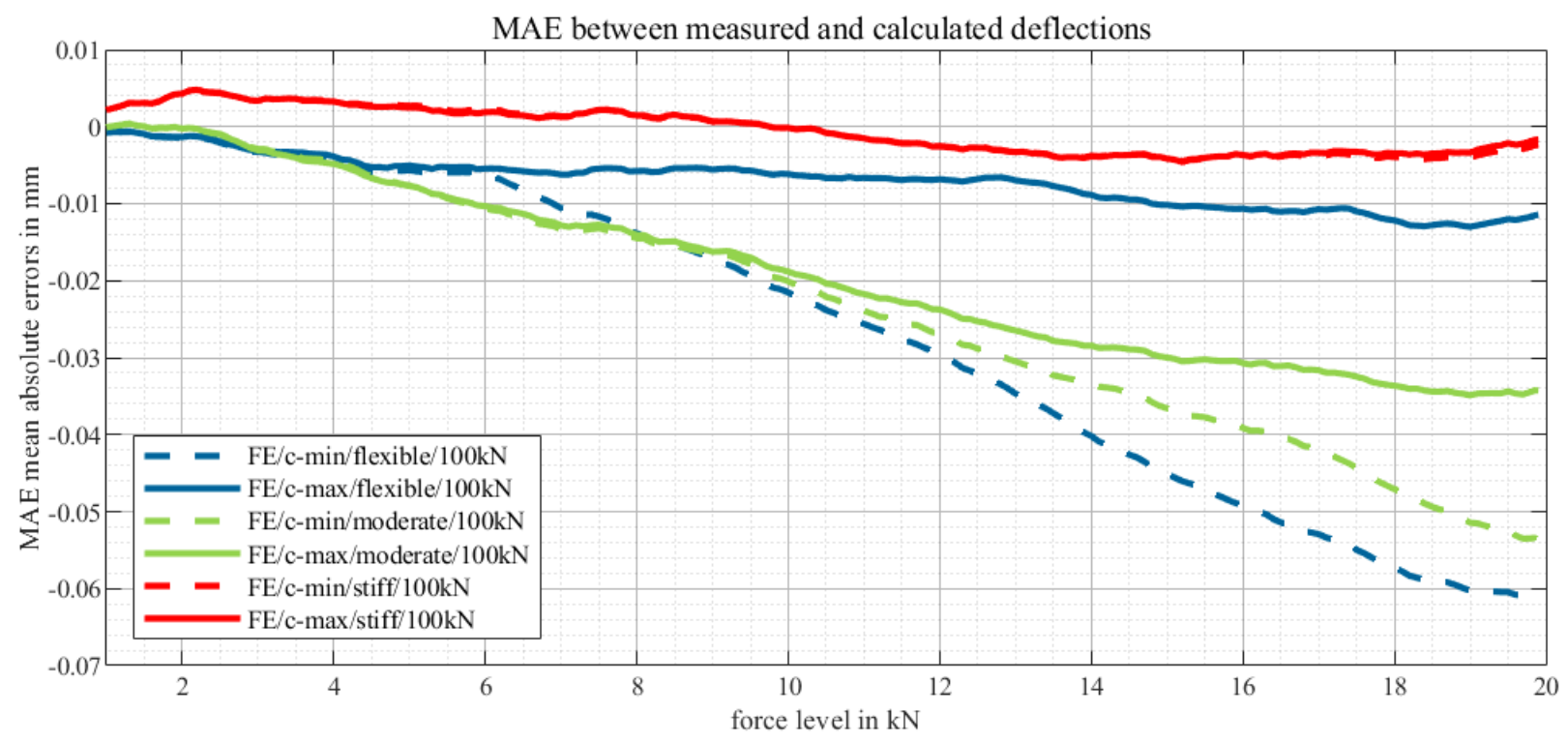

Figure 6: $M A E$ development over load level.

In order to investigate the overall load over deflection behaviour of the lower shaft, represented by the deflection at the centre of the roll set in $\mathrm{x}$-direction, the vertical load range for the FE simulation reaches a level of up to $70 \mathrm{kN}$. The experimental data are only up to a load level of $20 \mathrm{kN}$ and are not displayed in Fig. 7. As the simulations are displacement and not force controlled, the maximum force varies for each simulation. The movement of the upper against the lower shaft is $2 \mathrm{~mm}$ for the flexible and moderate roll sets and $1 \mathrm{~mm}$ for the stiff roll set. Independent from the clearances or the roll sets, the start-stiffness is almost linear (defined as the stiffness between $0 \mathrm{kN}$ and $20 \mathrm{kN}$ ) and the end-stiffness as well (defined as the stiffness between $36 \mathrm{kN}$ and the maximum of the occurring force level). 


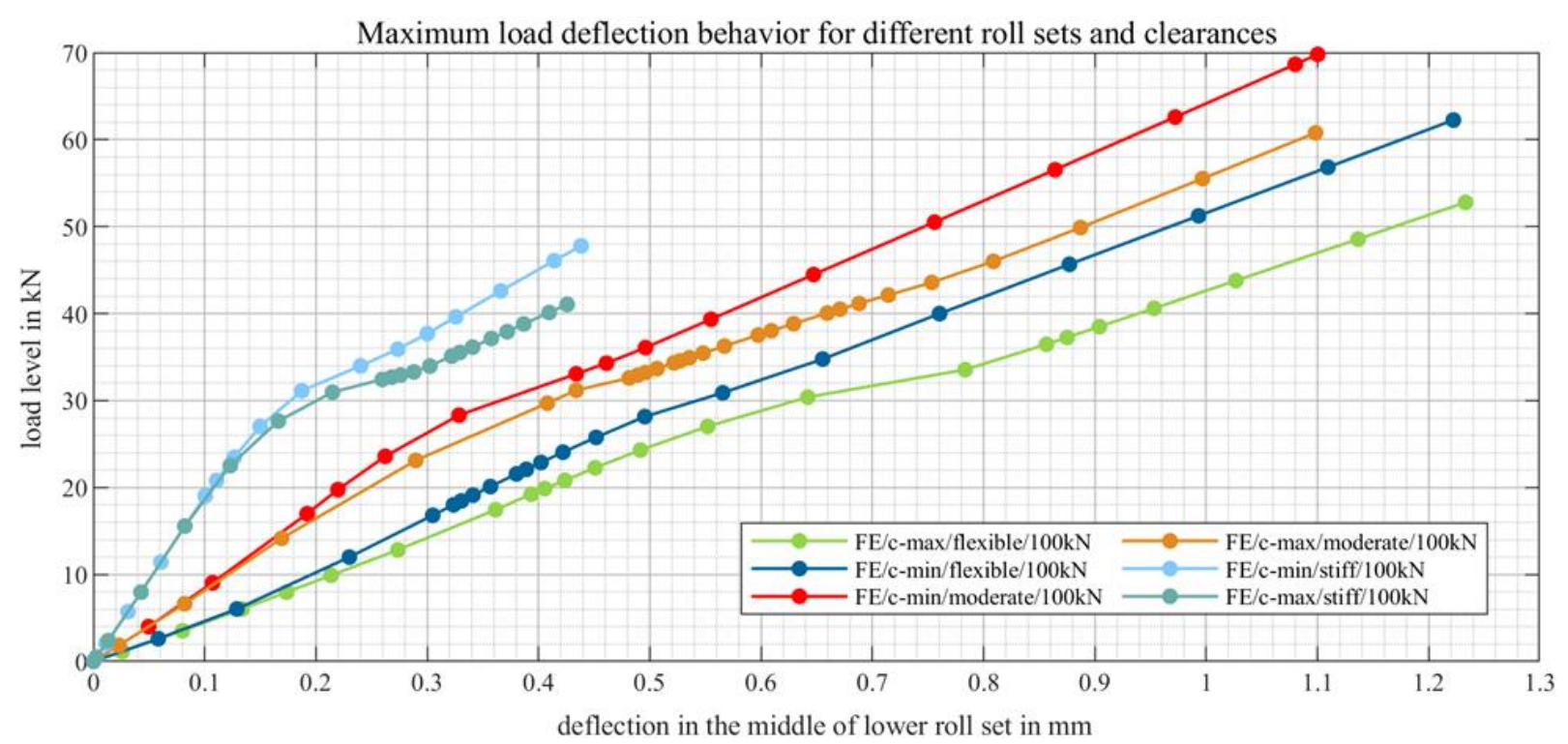

Figure 7: Load over deflection behaviour at the roll set's central position.

In Fig. 8 the start- and end-stiffnesses for the flexible/moderate/stiff roll set for minimum and maximum clearance are displayed. The end-stiffness is always smaller than the startstiffness, so by increasing the vertical load over the upper limit of the first linear region, the stiffness decreases. As one would expect, all simulations with maximum clearances are more compliant than the one with minimum clearances. The roll set itself for these three variations has the most impact on the stiffness. When considering the force-displacement curve, the question arises why there is a change in stiffness and thus a non-linearity.

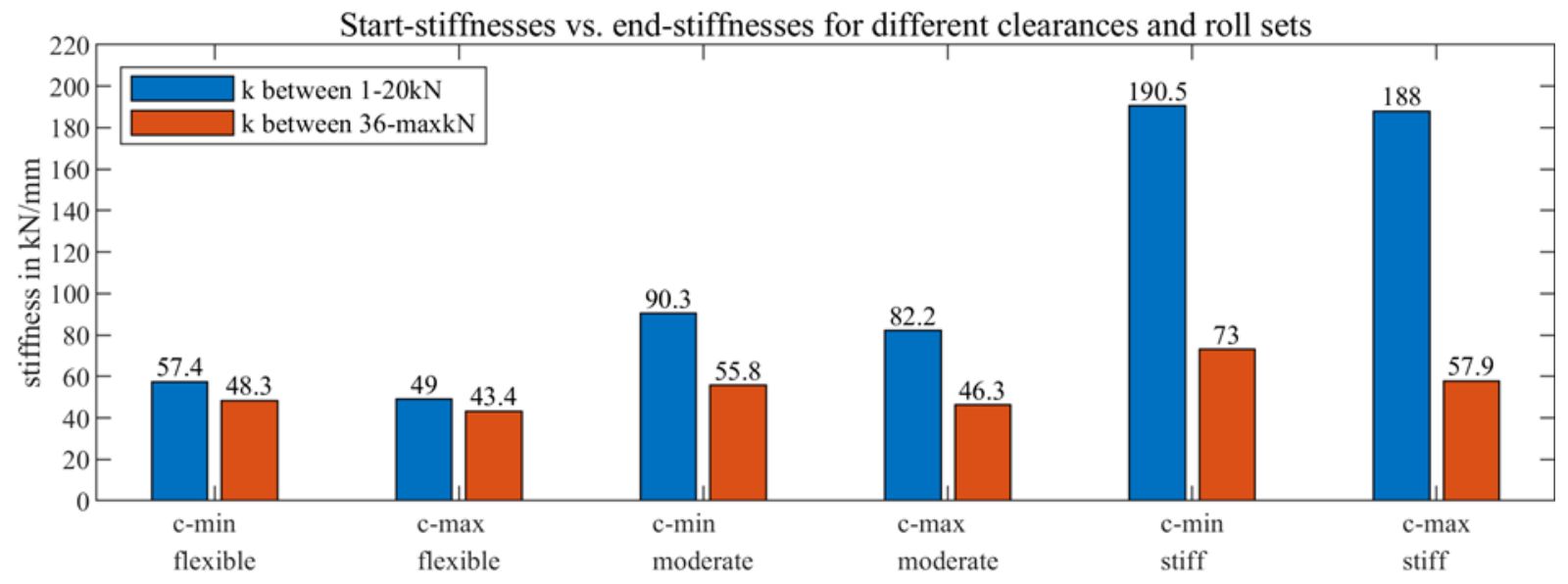

Figure 8: Start- vs. end-stiffnesses.

Fig. 9 deals with a more detailed consideration of the nonlinear load-deflection behaviour of the moderate roll sets with c-min and c-max. The diagram on the left shows the tilting angle of the bearing pedestal on the drive-side and the angle of inclination (or tilting angle) between point $\mathrm{A}$ and $\mathrm{B}$ of the shaft. The diagram on the right displays the support reactions at the drive side $\mathrm{A}, \mathrm{B}$ and the reaction force RF which is the sum of A and $\mathrm{B}$ as well as the support reaction at $\mathrm{C}$, which is identical to the drive-side reaction force RF. The force direction at the fixed bearing at A changes its direction due to the operation clearances of the bearings. As soon as the lower shaft is under load, the angular contact ball bearing gets in contact first, because its operation clearance is smaller compared to the needle bearing. This leads to tilting of the bearing pedestal counter clockwise, which in turn causes an immediate contact of the needle bearing. These phenomena occur during the second load step, in which gravity load is applied. 
Both bearings are in contact at the beginning of the last load step, which is displayed in the figures. Starting from the third load step the curves of the tilting angles of the shaft and pedestal are almost parallel, which means that the support force at B as well as at A increases. This can be observed up to a load level of about $17 \mathrm{kN}$ for c-min and about $29 \mathrm{kN}$ for c-max. This load region is also the maximum of the support force at $\mathrm{A}$ and at a load level of about $33 \mathrm{kN}$ for cmin and $44 \mathrm{kN}$ for c-max the support reaction in A is zero and gets negative from this point on. For higher force levels, the absolute values of the support forces at A and B increase linear and additionally the tiling behaviour becomes linear as well. The load range between $17 \mathrm{kN}$ to $33 \mathrm{kN}$ and $29 \mathrm{kN}$ to $44 \mathrm{kN}$ is also the nonlinear overall stiffness region (compare to Fig. 7).
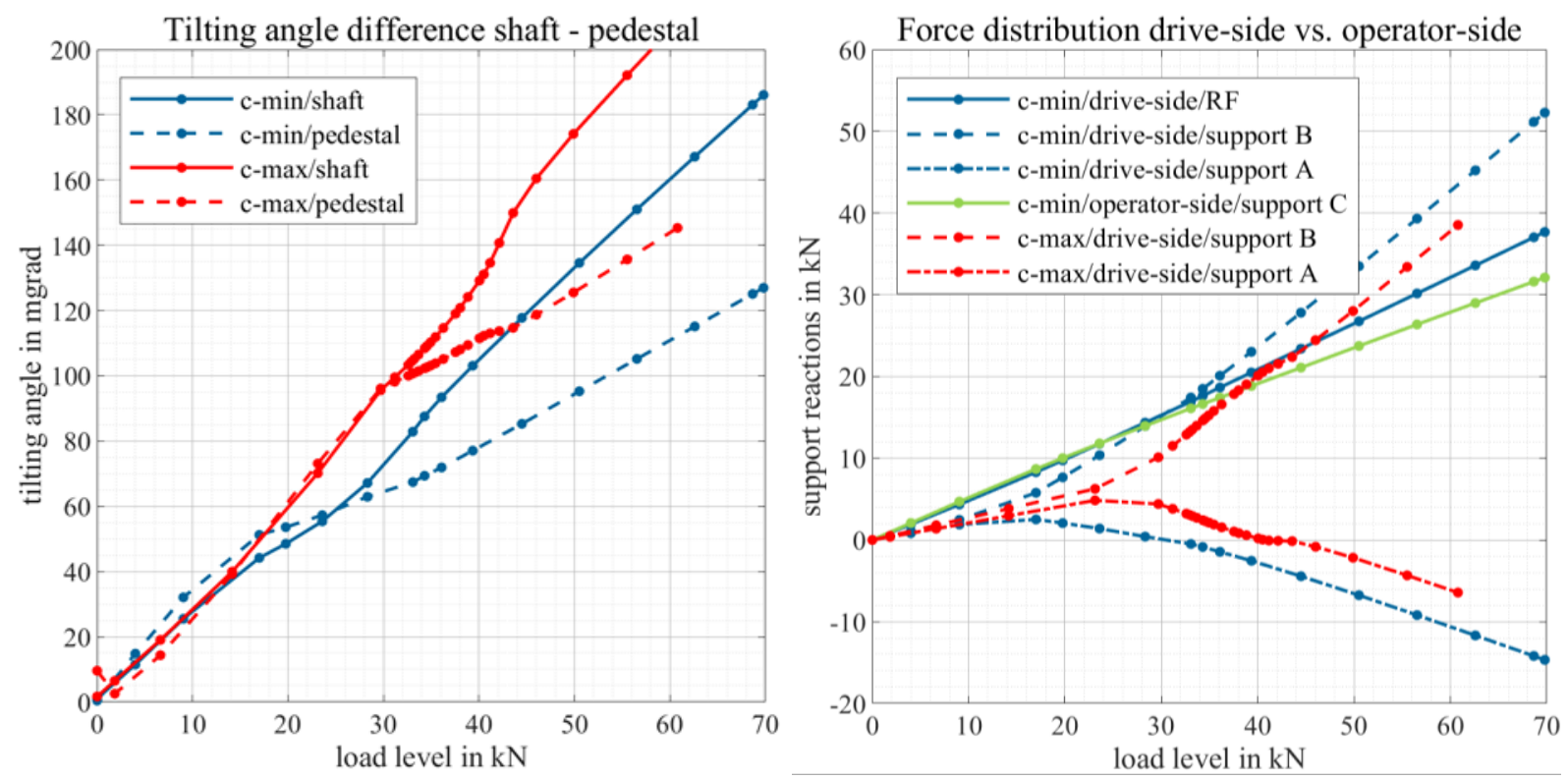

Figure 9: Tilting behaviour and support reactions.

\section{CONCLUSION}

The results of the load-deflection curves of the developed finite element model for the shaft with equipped rolls shows high accuracy compared with the experimental results. The mean absolute error $(M A E)$ over the load level for the simulation data with the corresponding experimental data (up to $20 \mathrm{kN}$ ) was calculated. Independent from the roll set, the results with maximum clearances show a minor error compared to the results with minimum clearances. This allows the assumption, that in reality the clearances are near the upper tolerance limit. All stiff roll sets and the flexible roll set with maximum clearance show a maximum absolute error of $0.01 \mathrm{~mm}$ over the full load range. All moderate roll sets and the flexible roll set with minimum clearances show errors increasing with rising load level, but no clear tendency of the variation parameters is visible. The maximum absolute $M A E$ can be observed for the flexible roll set with minimum clearances and adds up to $0.06 \mathrm{~mm}$. In summary, the finite element model is suitable to predict the load-deflection behaviour for these three roll sets.

Further investigations are needed to represent a wide range of different roll sets, with different geometries and quantities of rolls. A targeted variation of geometric parameters is necessary in order to make a general statement whether the model is accurate and reliable enough.

This study also sheds light on the sources of nonlinear behaviour occurring at higher load levels. Independent from the variation parameters, all simulation results show a nearly linear load-deflection behaviour at the centre of the roll sets, at the beginning and the end of the load range. The linearized start-stiffness $(1-20 \mathrm{kN})$ and end-stiffness $(36-\max \mathrm{kN})$ was calculated. 
As expected, the roll set has the highest impact on the start-stiffness, followed by the clearances. It is presumed that a main source of the nonlinear behaviour between the linear ranges, is the operating clearances of the bearings on the drive-side. The end of the first linear range is marked by the maximum positive support force at the angular contact ball bearing, which shifts to higher loads for maximum clearances compared to minimum clearances. The stiffness decreases up to the point, where the support force gets zero, continuing with a linear increase of the load causing a linear stiffness behaviour.

At a certain vertical load level, the rolls lose contact in the "tension" zone of the bending, which also leads to a different stiffness behaviour. Therefore, in future works, this fact has to be examined more closely.

The simulation showed the given interrelationships, although it seems to be almost impossible to investigate them in reality (accessibility of the measuring points, influence free measurements, etc.). The given explanations and coherences seem to be detailed but essentially affect the behaviour of the whole setup. They can be studied relatively easy in the presented model. In order to verify the validity of the model for the wide range of roll geometries, further experiments should be carried out and compared with the corresponding simulations.

\section{ACKNOWLEDGEMENT}

We thank ABAQUS for providing the software license.

\section{REFERENCES}

[1] Halmos, G. T. (2006). Roll Forming Handbook, CRC Press, Boca Raton

[2] Sáenz de Argandoña, E.; Larrañaga, J.; Legarda, A.; Galdos, L. (2012). Roll forming set-up influence in the forming forces and profile quality, Key Engineering Materials, Vol. 504-506, 1249-1254, doi:10.4028/www.scientific.net/KEM.504-506.1249

[3] Jurkovic, M.; Jurkovic, Z.; Buljan, S.; Obad, M. (2018). An experimental and modelling approach for improving utilization rate of the cold roll forming production line, Advances in Production Engineering \& Management, Vol. 13, No. 1, 57-68, doi:10.14743/apem2018.1.273

[4] Traub, T.; Miks, C.; Groche, P. (2017). Force measurements supporting the set-up process in roll forming, ATINER'S Conference Paper Series, Paper MEC2017-2346, 21 pages

[5] Kim, B. S.; Kim, T. G. (2019). Cooperation of simulation and data model for performance analysis of complex systems, International Journal of Simulation Modelling, Vol. 18, No. 4, 608-619, doi:10.2507/IJSIMM18(4)491

[6] Vieira, A. A. C.; Dias, L. M. S.; Santos, M. Y.; Pereira, G. A. B.; Oliveira, J. A. (2018). Setting an Industry 4.0 research and development agenda for simulation - a literature review, International Journal of Simulation Modelling, Vol. 17, No. 3, 377-390, doi:10.2507/IJSIMM17(3)429

[7] Lindgren, M. (2009). Experimental and Computational Investigation of the Roll Forming Process, $\mathrm{PhD}$ Thesis, Luleå University of Technology, Luleå

[8] Mohammdi Najafabadi, H.; Moslemi Naeini, H.; Safdarian, R.; Kasaei, M. M.; Akbari, D.; Abbaszadeh, B. (2018). Effect of forming parameters on edge wrinkling in cold roll forming of wide profiles, The International Journal of Advanced Manufacturing Technology, Vol. 101, No. 1-4, 181-194, doi:10.1007/s00170-018-2885-x

[9] Paralikas, J.; Salonitis, K.; Chryssolouris, G. (2009). Investigation of the effects of main rollforming process parameters on quality for a V-section profile from AHSS, The International Journal of Advanced Manufacturing Technology, Vol. 44, No. 3-4, 223-237, doi:10.1007/s00170008-1822-9

[10] Rossi, B.; Degée, H.; Boman, R. (2013). Numerical simulation of the roll forming of thin-walled sections and evaluation of corner strength enhancement, Finite Elements in Analysis and Design, Vol. 72, 13-20, doi:10.1016/j.finel.2013.04.002 
[11] Tsang, K. S.; Ion, W.; Blackwell, P.; English, M. (2017). Validation of a finite element model of the cold roll forming process on the basis of 3D geometric accuracy, Procedia Engineering, Vol. 207, 1278-1283, doi:10.1016/j.proeng.2017.10.883

[12] Wiebenga, J. H.; Weiss, M.; Rolfe, B.; van den Boogaard, A. H. (2013). Product defect compensation by robust optimization of a cold roll forming process, Journal of Materials Processing Technology, Vol. 213, No. 6, 978-986, doi:10.1016/j.jmatprotec.2013.01.006

[13] Abeyrathna, B.; Rolfe, B.; Hodgson, P.; Weiss, M. (2016). A first step towards a simple in-line shape compensation routine for the roll forming of high strength steel, International Journal of Material Forming, Vol. 9, 423-434, doi:10.1007/s12289-015-1238-7

[14] Traub, T.; Chen, X.; Groche, P. (2017). Experimental and numerical investigation of the bending zone in roll forming, International Journal of Mechanical Sciences, Vol. 131-132, 956-970, doi:10.1016/j.ijmecsci.2017.07.056

[15] Müller, C. (2014). Numerische Abbildung und Validierung von Beanspruchungsgrößen in Rollprofilierprozessen, PhD Thesis, Technische Universität Darmstadt, Darmstadt

[16] Lamprecht, M.; Kocbay, E.; Leonhartsberger, M.; Vetyukov, Y.; Bleicher, F. (2021). Nonlinear mechanical model of the shaft of a roll forming mill and parameter identification, The International Journal of Advanced Manufacturing Technology, Vol. 112, No. 11-12, 3363-3375, doi:10.1007/ s00170-020-06412-5

[17] Leonhartsberger, M.; Lamprecht, M.; Bleicher, F. (2020). Influence parameters on tool deflections in roll forming, Proceedings of the $31^{\text {st }}$ DAAAM International Symposium, 0701-0707, doi:10.2507/31st.daaam.proceedings.097

[18] Sanchez-Marin, F.; Roda-Casanova, V.; Porras-Vazquez, A. (2018). A new analytical model to predict the transversal deflection under load of stepped shafts, International Journal of Mechanical Sciences, Vol. 146-147, 91-104, doi:10.1016/j.ijmecsci.2018.07.023

[19] Radi, E.; Lanzoni, L.; Strozzi, A.; Bertocchi, E. (2017). Shaft-hub press fit subjected to bending couples: Analytical evaluation of the shaft-hub detachment couple, Applied Mathematical Modelling, Vol. 50, 135-160, doi:10.1016/j.apm.2017.05.018

[20] Dassault Systemes (2014). Abaqus 6.14: Abaqus/CAE User's Guide, Dassault Systemes, VélizyVillacoublay 